\title{
Friedrich Schleiermacher, Conférences sur l'éthique, la politique et l'esthétique (1814-1833)
}

(Textes traduits, présentés et annotés par Jean-Marc Tétaz). Genève, Labor et Fides, coll. « Les classiques du christianisme moderne », 2011, $464 \mathrm{p}$.

Daniel Vidal

\section{(2) OpenEdition}

Édition électronique

URL : http://journals.openedition.org/assr/24725

DOI : $10.4000 /$ assr. 24725

ISSN : $1777-5825$

Éditeur

Éditions de l'EHESS

\section{Édition imprimée}

Date de publication : 30 décembre 2012

Pagination : 280

ISSN : 0335-5985

\section{Référence électronique}

Daniel Vidal, « Friedrich Schleiermacher, Conférences sur l'éthique, la politique et l'esthétique

(1814-1833) », Archives de sciences sociales des religions [En ligne], 160 | octobre-décembre 2012, mis en ligne le 08 avril 2013, consulté le 21 septembre 2020. URL : http://journals.openedition.org/assr/ 24725 ; DOI : https://doi.org/10.4000/assr.24725 


\section{Friedrich Schleiermacher, Conférences sur l'éthique, la politique et l'esthétique (1814-1833)}

(Textes traduits, présentés et annotés par Jean-Marc Tétaz). Genève, Labor et Fides, coll. « Les classiques du christianisme moderne », 2011, $464 \mathrm{p}$.

Daniel Vidal

\section{RÉFÉRENCE}

Friedrich Schleiermacher, Conférences sur l'éthique, la politique et l'esthétique (1814-1833), (Textes traduits, présentés et annotés par Jean-Marc Tétaz). Genève, Labor et Fides, coll. «Les classiques du christianisme moderne », 2011, $464 \mathrm{p}$. 
1 Après ses années de prédications inspirées de son entourage morave, et la publication de son Discours sur la religion (1799), puis son professorat à Halle de 1804 à 1807 et la publication en 1803 de sa Critique de tous les systèmes de morale, F. Schleiermacher (1768-1834) est nommé professeur de théologie à Berlin, de 1810 à 1833, où il prononcera les douze Conférences sur l'éthique, le droit, l'État et l'esthétique. De la «construction scientifique » de la question éthique à son accomplissement en l'esthétique, ce "Bien suprême ", ces conférences réunies et commentées aujourd'hui par J.-M. Tétaz, accompagnent, et explicitent, une œuvre dissidente par rapport à l'héritage de Kant et de Fichte. Dans son Discours sur la religion, et, dans le contexte des années berlinoises où il publie son traité sur La foi chrétienne d'après les principes de la Réforme (1821-1822), en fondant la religion sur l'intuition personnelle et en définissant la foi comme "province particulière de la sensibilité " face à l'infiniment Grand et Bien, Schleiermacher avait bouleversé la pensée théologique en son point névralgique : le moment central du « sentiment religieux » est cette "piété du cœur", cette "présence éprouvée de l'absolu en nous", selon la formule de P.Demange, que seule peut autoriser une expérience personnelle, existentielle. Si je rappelle ce contexte, sa familiarité avec le romantisme allemand (fréquentation des cercles autour de Novalis et Schlegel), et cette dissidence théologique opposant l'Église authentique à l'Église institutionnelle, c'est pour souligner le caractère parfaitement novateur des études déployant l'éthique jusqu'à son noyau de "vérité ", l'esthétique, et son fondement chaque fois singulier. En tant que telles, ces douze études trouvent leurs assises et leurs références dans cette expérience intime qui conduit le philosophe à affirmer qu'« une religion sans Dieu peut être meilleure qu'une autre avec Dieu», et qu'une conception du Bien ne saurait être une "téléologie naturelle de l'être humain », ainsi que le résume fortement J.-M. Tétaz, mais doit s'expliciter en une «éthique de l'autonomie».

Il fallait, pour cela, se situer délibérément dans l'après-Kant. Si le devoir comme raison du bien ne peut rendre compte de la sphère éthique en son ensemble, c'est parce que l'impératif catégorique "ne peut être au principe de motivations de l'action bonne». Allons plus loin : purement « formelle » et garantissant ainsi chez Kant son universalité (coercition intérieure, mais intellectuelle), la loi morale, pour Schleiermacher, résumé par J.-M. Tétaz, « exclut qu'elle soit le principe de la matérialité des actions éthiques ». À l'inverse de toute conception catégorique de la loi morale, il convient, remarque le commentateur, de « reconstruire le sens de la vie humaine comme vie consciente de soi ». Où l'on retrouve l'intuition au principe de l'immersion religieuse, cette «intuition de soi », dont traite J.-M. Tétaz, «dans laquelle la vie consciente de soi se saisit comme la réalisation individuelle de la raison et se forme selon la loi individuelle ». La vie est action. La vie éthique sera donc, à raison de la structure complexe, de l'agir. L'agir : cet "acte concret » effectué " en vue d'une totalité », un «horizon de sens" (le "Bien suprême») - mais soumis à l'instance cruciale de la 
« décision » prise en sa toute singularité. C'est dire que dans l'éthique, agir est vouloir. Et si tout acte est bien "œuvre », et donc modification du monde, en même temps que "présentation », et par là «manifestation de l'intériorité », l'agir concret/singulier est toujours immédiatement intersubjectif.

Il est remarquable que, une fois délestée de son impératif catégorique universel, l'éthique de l'action institue le sujet singulier au centre de la trame qui relie devoir, vertu, sagesse, loi morale, bien suprême. L'éthique de l'action, chez Schleiermacher, est la «quintessence» de l'identique et du spécifique - de l'universel, et de ce que le philosophe nomme «l'imagination ». À la production de savoirs, conformément au respect de normes et de règles à valeur universelle, répond la capacité de solliciter librement les "principes de réalisation individuelle", selon la diversité des êtres humains. Chaque acte est accomplissement de soi dans l'horizon partagé de la communauté. Chaque communauté aussi bien s'accomplit en chacun de ses constituants. Et Schleiermacher propose cela, qui n'en finira pas de surprendre kantiens, héritiers et épigones, et que définit ainsi J.-M. Tétaz : «l'œuvre d'art est le paradigme du Bien suprême », et la production artistique, le "paradigme de l'activité éthique ». On voit l'enjeu de cette nouvelle conception éthique, ainsi qu'il en va de la philosophie : cheminement «en commun vers le savoir, plutôt que déploiement d'un savoir d'ores et déjà acquis ». Car rien n'est déjà là en l'action éthico-artistique, mais tout est à re-fonder, ré-inventer, dans le cadre d'une décision « dont la plausibilité est indépendante de sa déduction métaphysique ».

4 En ce sens, l'imagination est cette force vitale qui rebat sans cesse les cartes de la loi, hors de toute saisie définitive, et de toute possession "scientifique", mais comme « approche infinie de l'Absolu ». Si Kant et Fichte échouent à « saisir la vie humaine en son entier ", sans doute doit-on alors, commente J.-M. Tétaz, récuser l'impératif catégorique comme paradigme de l'éthique, et «le remplacer par celui de l'art: le modèle fondamental du Bien suprême, c'est le monde comme œuvre d'art », sa perfection, son accomplissement. Mais le Bien est horizon de l'action, et non sa "fondation ultime». Nulle transcendance, ici, et nulle position métasociale. Car l'action, on l'a vu, est transformation du "monde ", loin d'en être la totalisation. Parce qu'elle est ouverture, et toujours un acte par principe individuel, selon une règle engagée dans une perspective plus large. Règle: l'artiste, il va de soi, exécute une œuvre en fonction d'un modèle intime, ce que Schleiermacher appelle "un archétype ». Mais ce modèle ne lui impose aucune prescription quant à l'ordre à suivre. Cela ressortit de sa seule et intime décision. De son "vouloir», cette condition au fondement de l'éthique. Et de la liberté.

5 Si l'éthique doit s'entendre comme discipline scientifique capable de rendre compte de l'action humaine, encore faut-il questionner sa structure sémantique, et mettre de l'ordre "par rapport au langage quotidien » et sa confusion. Schleiermacher contre, aujourd'hui, Wittgenstein. Et par exemple, inscrire la sémantique de la loi, résume J.M. Tétaz, "dans le cadre d'une métaphysique de la nature et de la raison », qui est de constitution anthropologique. Telle loi, morale/naturelle, et telle éthique qui en découle, échappent à la fonction kantienne d'impératif catégorique et universel. Le Bien suprême, dès lors, est cette "réalité éthique différenciée ", qui rend compte de «l'ensemble vivant de tout ce qui procède de la volonté raisonnable ». Et qui autorise le déploiement de l'individualité, cette "déterminité contingente », considérée comme "dimension constitutive de l'être-homme ». La théorie de l'action éthique inscrit tout 
«acte concret», et particulier, dans le cadre de la «socialité », et son résultat, irréductible à toute volonté ou observance d'un devoir. L'action éthique porte modification du monde, ouvert dès lors à son aventure. Par elle, en effet, se conjuguent expérience personnelle et existentielle d'un sujet singulier, et structure d'accueil d'une " communauté » où cette action se déploie. La communauté, peut alors écrire J.-M. Tétaz, «est le sujet de l'action éthique » en tant qu'elle reçoit l'individu «avec sa volonté éthique originelle ».

6 La perfection du « bien suprême » n'est pas l'État kantien, mais, on l'a vu, la perfection esthétique, qui rassemble tous les réquisits de l'éthique. Ce Bien est-il l'accomplissement de la loi ? Non, dit Schleiermacher, mais, selon la formule de J.M. Tétaz, un enchaînement de "négations successives du mouvement de la pensée », et donc son impossible clôture, "seule façon, précise le commentateur, dont puisse être indirectement représenté l'absolu». Si bien que l'œuvre d'art est, par définition, toujours ouverte à plus encore de projets et d'histoire. Et l'éthique qui s'y énonce est en permanence remise en jeu et en doute, toujours alors réinventée. Car l'esthétique de Schleiermacher distingue radicalement l'art d'avec le beau, ou le sublime, ou, plus encore, l'imitation de la nature, ou sa contemplation. Si le Bien suprême est l'art, c'est précisément parce que l'art est l'achèvement formel d'une œuvre «close sur ellemême", toutes symbolisations intérieures assumées par l'artiste, et tout affect maîtrisé. Cette maîtrise est indispensable au déploiement global de l'imagination, cette force éthique qui refonde en permanence la loi. Seule l'imagination s'oppose aux manifestations spontanées de l'affect, et "s'intercale» entre cet affect et son expression, qu'elle bloque. Par réflexion, pondération, elle relève pleinement de l'éthique de l'action, et permet la conjugaison d'une disposition affective individuelle, autonome, cette «tonalité fondamentale de l'existence ", et le recours à des ressources symboliques collectives, dont chacun s'approprie singulièrement la tension et la charge cognitive. Par là, l'imagination artistique est d'emblée, souligne J.-M. Tétaz, une "dimension intersubjective». Cette intersubjectivité est ainsi la figure centrale et aboutie d'une éthique qui pose le sujet, et la conscience de soi, comme condition de la communauté, «l'artiste comme interprète du public auquel il donne voix », et l'action comme re-création continue du monde comme « œuvre d'art ». 